\title{
Características da carne de tourinhos Red Norte suplementados com óleos de fritura e soja terminados em confinamento
}

\author{
Meat characteristics of Red Norte young bulls supplemented with fry and soybeans oil \\ finished in feedlot
}

\author{
RODRIGUES FILHO, Moacir ${ }^{1}$; PÉRES, Juan Ramon Olalquiaga ${ }^{2}$; RAMOS, Eduardo \\ Mendes $^{3}$; RODRIGUES, Nair Elizabeth Barreto ${ }^{1}$; LOPES, Leandro Sâmia ${ }^{4 *}$
}

\footnotetext{
${ }^{1}$ Instituto Federal do Espírito Santo, Departamento de Zootecnia, Santa Tereza, Espírito Santo, Brasil.

${ }^{2}$ Universidade Federal de Lavras, Departamento de Zootecnia, Lavras, Minas Gerais, Brasil.

${ }^{3}$ Universidade Federal de Lavras, Departamento de Ciências dos Alimentos, Lavras, Minas Gerais, Brasil.

${ }^{4}$ Universidade do Estado de Santa Catarina, Departamento de Zootecnia, Chapecó, Santa Catarina, Brasil.

*Endereço para correspondência: leandrosamia@uol.com.br
}

\section{RESUMO}

Objetivou-se com este trabalho avaliar o efeito da suplementação lipídica na terminação de tourinhos Red Norte sobre as características qualitativas da carne. Foram utilizados 27 tourinhos com peso corporal inicial médio de $317 \pm 33 \mathrm{~kg}$, submetidos as seguintes dietas: sem suplementação lipídica (SSL); suplementados com óleo de soja (SOS) e suplementados com óleo de fritura (SOF). A duração do experimento foi de 112 dias, sendo 28 dias de adaptação e 84 dias experimentais. Os animais receberam dieta "ad libitum", para ganho médio diário de $1,5 \mathrm{~kg}$ e foram abatidos em frigorífico industrial. A composição centesimal e o colesterol da carne, e da gordura subcutânea não foram influenciadas pelos tratamentos $(\mathrm{P}>0,05)$. Houve interação $(\mathrm{P}<0,01)$ entre tratamento $\mathrm{e}$ dias para força de cisalhamento (FC) enquanto que para as características de capacidade de retenção de água (CRA), perda por cozimento (PPC) e $\mathrm{pH}$ houve apenas efeito de tratamento $(\mathrm{P}<0,01)$. Para as características de cor, houve interação entre tratamento e dia para a intensidade de amarelo $\left(b^{*}\right)$, índice de saturação $\left(\mathrm{c}^{*}\right)$ e ângulo de tonalidade $\left(\mathrm{h}^{*}\right) \quad(\mathrm{P}<0,5)$, enquanto que para intensidade de vermelho $\left(\mathrm{a}^{*}\right)$ houve efeito de tratamento $(\mathrm{P}<0,001)$ e dias $(\mathrm{P}<0,001)$ e para luminosidade $\left(\mathrm{L}^{*}\right)$ houve efeito apenas de tratamento $(\mathrm{P}<0,001)$. A suplementação lipídica não influenciou a composição centesimal da carne e o seu teor de colesterol. O óleo de soja influenciou a cor da carne, sem comprometer sua qualidade. Já o óleo de fritura apresentou uma carne com elevado $\mathrm{pH}$, o que é indesejável para o consumo humano.

Palavras-chave: bovino, maturação, qualidade de carne, suplementação lipídica

\section{SUMMARY}

The objective of this study was to evaluate the effect of the lipid supplementation on finishing of Red Norte young bulls in the meat qualitative characteristics. Twenty seven young bulls with at initial average weight of $317 \pm 33 \mathrm{~kg}$ were used, according with the diets: no lipid supplementation (SSL), supplemented with soybeans oil (SOS) and supplemented with fry oil (SOF). The experiment lasted 112 days, being 28 days was for adaptation to experimental diets. The animals received " $a d$ libitum" diets to average daily gain of $1,5 \mathrm{~kg}$ and were slaughtered in industrial slaughterhouse. The chemical composition and the cholesterol of the meat and the subcutaneous fat were not influenced by treatments $(\mathrm{P}>0,05)$. There was interaction $(\mathrm{P}<0,01)$ between treatments and share force (FC) whereas for water holding capacity (CRA), cooking losses (PPC) and $\mathrm{pH}$ there was only treatment effect $(\mathrm{P}<0,01)$. To characteristics of color, there was interaction between treatment and day to yellow intensity $\left(b^{*}\right)$, saturation index $\left(\mathrm{c}^{*}\right)$ and tone angle $\left(\mathrm{h}^{*}\right)$ $(\mathrm{P}>0,05)$, whereas for red intensity $\left(\mathrm{a}^{*}\right)$ there was effect of treatment $(\mathrm{P}<0,001)$ and days 
$(\mathrm{P}<0,001)$ and to luminosity $\left(\mathrm{L}^{*}\right)$ there was effect only to treatment $(\mathrm{P}<0,001)$. The lipid supplementation was not influenced the chemical composition of the meat and its cholesterol content. The soybeans oil influenced the meat color, without compromising its quality. However, the fry oil showed meat with high $\mathrm{pH}$, which is undesirable for human consumption.

Keywords: cattle, lipid supplementation, maturation, meat quality

\section{INTRODUÇÃO}

O processo contínuo e irreversível de intensificação da pecuária nacional aliado a outros fatores mercadológicos tem alavancado a produção $\mathrm{e}$ aumentado às exportações brasileiras de carne bovina. Nesse cenário em curso a busca pela qualidade da carne a ser produzida e exportada passa a ser um fator determinante para consolidar $\mathrm{e}$ estabelecer novos mercados potenciais.

A qualidade da carne bovina é uma característica multifatorial e pode ser avaliada de forma objetiva e subjetivamente pelos seus atributos sensoriais, tecnológicos, sanitários e nutricionais, acrescida dos aspectos éticos e ambientais sobre os quais fora produzida.

Recentes estudos tem avaliado a utilização de fontes lipídicas na nutrição de bovinos confinamentos (OLIVEIRA, et al., 2011; RODRIGUES FILHO et al., 2013) como modificador da qualidade da carne. Entretanto algumas fontes lipídicas tem seu uso restrito devido ao seu elevado custo de aquisição. Sendo assim, a utilização de óleo de fritura, oriundo do processo de fritura por imersão, pode ser utilizado como fonte alternativa em substituição a fontes tradicionais de lipídeos, pois podem além de reduzir os custos de produção, podem também alterar a composição tecidual da carcaça e reduzir o impacto ambiental, devido ao destino incorreto deste resíduo no ambiente.

Considerado, ambientalmente, um agente poluidor dos sistemas fluviais, mananciais e da vida aquática, além de grandes prejuízos ao sistema de captação de esgoto urbano, o óleo de fritura vem sendo gerado em quantidades crescentes ser ter destinação própria na fonte geradora, e pode ser utilizado na alimentação animal contribuindo para a redução deste resíduo no ambiente.

O óleo de fritura é um resíduo de composição e cor variável dependendo da fonte, característica lipídica do alimento preparado, tempo e temperatura de utilização no processo de fritura. Entretanto, a magnitude e a persistência das modificações que este produto pode causar aos atributos quantitativos $\mathrm{e}$ qualitativos da carne de bovinos submetidos à suplementação com óleo de fritura ainda não são conclusivos, portanto, ainda demandam estudos.

Diante do exposto, objetivou-se com este trabalho avaliar as características qualitativas e diferentes tempos de maturação na carne de tourinhos Red Norte suplementados com óleos de fritura e de soja terminados em confinamento.

\section{MATERIAL E MÉTODOS}

O experimento foi conduzido no Setor de Bovinocultura do Departamento de Zootecnia da Universidade Federal de Lavras. Foram utilizados 27 tourinhos da raça Red Norte, com peso corporal inicial médio de $317 \pm 33 \mathrm{~kg}$ e idade média inicial de $19 \pm 1$ mês.

Os animais foram mantidos em regime de confinamento, em currais coletivos com área de $30 \mathrm{~m}^{2} /$ animal, separados aleatoriamente por tratamento. Os currais eram de piso de terra batida, sem 
cobertura e com divisórias feitas de arame liso. Os bebedouros eram coletivos localizados na divisória entre currais e o comedouro do tipo vinilona, disposto transversalmente na parte superior dos currais, com $70 \mathrm{~cm}$ lineares/animal.

Foram utilizadas três dietas experimentais: sem suplementação lipídica (SSL); suplementação com óleo de soja (SOS) e suplementação com óleo de fritura (SOF). O óleo de fritura era coletado semanalmente em restaurantes da cidade e armazenado na fabrica de ração em tambores de plástico com capacidade para 100 litros à temperatura ambiente.

As dietas com adição de lipídeo foram elaboradas semanalmente e formuladas para atingir um nível máximo de $7 \%$ de extrato etéreo (EE) na MS, e, atender as exigências de ganho de $1,5 \mathrm{~kg} / \mathrm{dia}$, de acordo com o NRC (2000) (Tabela 1) sendo fornecida diariamente ad libitum na forma de ração completa às $8 \mathrm{~h} 00$ e às $15 \mathrm{~h} 00$.

Tabela 1. Proporção dos ingredientes e composição química das dietas experimentais

\begin{tabular}{|c|c|c|c|}
\hline \multirow{3}{*}{ Ingredientes } & \multicolumn{3}{|c|}{ Tratamentos } \\
\hline & \multicolumn{3}{|c|}{ Proporção (\%MS) } \\
\hline & SSL & SOS & SOF \\
\hline Silagem de milho & 40,0 & 40,0 & 40,0 \\
\hline Milho integral moído & 50,4 & 46,8 & 46,8 \\
\hline Farelo de soja & 7,8 & 7,8 & 7,8 \\
\hline Óleo de soja & 0,0 & 3,6 & 0,0 \\
\hline Óleo de fritura & 0,0 & 0,0 & 3,6 \\
\hline Núcleo mineral & 1,8 & 1,8 & 1,8 \\
\hline Nutrientes & \multicolumn{3}{|c|}{ Composição química (\%) } \\
\hline Matéria seca $^{1}$ & 48,5 & 47,5 & 46,9 \\
\hline Proteína bruta $^{2}$ & 11,4 & 11,2 & 11,0 \\
\hline Fibra em detergente neutro $\left(\mathrm{FDN}_{\mathrm{CP}}\right)^{2}$ & 32,5 & 31,8 & 31,4 \\
\hline Carboidratos não fibrosos ${ }^{2}$ & 49,0 & 45,9 & 46,2 \\
\hline Extrato etéreo $^{2}$ & 3,4 & 7,1 & 7,3 \\
\hline Nutrientes digestíveis totais ${ }^{2,}$ & 70,9 & 77,4 & 78,2 \\
\hline
\end{tabular}

${ }^{\mathrm{T}}$ Base da matéria natural; ${ }^{2}$ Base da matéria seca.

O período de confinamento foi composto de 28 dias de adaptação às dietas, e 84 dias de período experimental. Para a obtenção do peso corporal inicial e final, os animais foram submetidos a jejum alimentar e hídrico de 16 horas.

Um dia antes de serem levados ao frigorífico os animais foram pesados, após jejum de sólidos de 16 horas. No dia seguinte, foram transportados em caminhão boiadeiro para um frigorífico industrial distante $60 \mathrm{~km}$. No frigorífico, durante o pré-abate, os animais foram submetidos a jejum de sólidos e líquidos por 24 horas em currais sem cobertura. Os animais foram abatidos com 481,0; 488,7 e $462,6 \mathrm{~kg}$ de PV e apresentaram 251,$9 ; 251,6$ e $243,8 \mathrm{~kg}$ de carcaça para os tratamentos sem suplementação lipídica, suplementados com óleo de soja e suplementados com óleo de fritura respectivamente.

$\mathrm{O}$ abate dos animais foi realizado de acordo com as normas do RIISPOA (BRASIL, 1997), sob inspeção federal. 
Os animais foram insensibilizados por concussão cerebral e sangria, seguindose, a esfola, evisceração, divisão das carcaças e resfriamento. As meiascarcaças foram resfriadas, a $1^{0} \mathrm{C}$ por 48 horas, até o momento da coleta das amostras.

Para a avaliação da composição centesimal e de colesterol foi retirada uma amostra do músculo Longíssimus dorsi, entre a $12^{\mathrm{a}}$ e $13^{\mathrm{a}}$ costelas da meia carcaça resfriada. As amostras foram embaladas e congeladas. Para a realização da análise centesimal, as amostras foram descongeladas e a gordura subcutânea foi previamente removida. Uma amostra de $100 \mathrm{~g}$ de carne foi picada em cubos de $0,5 \mathrm{~cm}$, e levada à estufa com circulação forçada a 55 a $60^{\circ} \mathrm{C}$ por 72 horas para présecagem. Em seguida a amostra foi resfriada, moída em moinho tipo Willey com peneira de $1 \mathrm{~mm}$, e armazenadas para análises, onde a determinação da $\mathrm{PB}$, EE, MM e MS seguiram as metodologias da AOAC (2005).

A extração dos lipídeos do músculo (gordura intramuscular) e da gordura subcutânea para determinação do colesterol foi realizada de acordo com Folch et al. (1957). Amostras de 5g foram moídas e homogeneizadas em $50 \mathrm{~mL}$ de solução clorofómio/metanol (2:1), e filtradas em papel de filtro, para funil de separação de $500 \mathrm{~mL}$. O material filtrado foi agitado com $10 \mathrm{~mL}$ de solução de cloreto de potássio a $12 \%$, onde permaneceu em repouso por 2 horas para separação das porções polar e apolar. A porção polar foi descartada e a porção apolar foi submetida à nova separação, iniciada com agitação de $6 \mathrm{~mL}$ de solução de cloreto de potássio, onde permaneceu em repouso por 12 horas. Após a segunda separação, a fração apolar foi recolhida para um balão volumétrico de $50 \mathrm{~mL}$, ao qual foi adicionado clorofórmio até completar o volume. Foi retirado uma amostra de $5 \mathrm{~mL}$ para determinação do colesterol. $\mathrm{O}$ colesterol foi determinado por colorimetria, de acordo com Bragagnolo \& Rodriguez-Amaya (1995).

Para a avaliação da cor, pH, CRA, PPC, e da força de cisalhamento (FC), nos tempos 0,7 e 14 dias, foram extraídos três amostras de $2,5 \mathrm{~cm}$ de espessura do músculo Longissimus dorsi entre a $12^{\mathrm{a}} \mathrm{e}$ $13^{\mathrm{a}}$ costelas da meia carcaça resfriada. As amostras foram identificadas $\mathrm{e}$ embaladas em sacos de polietileno de alta resistência a vácuo.

As amostras submetidas ao processo de maturação foram armazenadas à temperatura constante de $2^{0} \mathrm{C}$, em geladeira com controle de temperatura. As três amostras de cada animal, foram distribuídas nos três tempos de avaliação: 0, 7 e 14 dias de maturação, após o resfriamento das carcaças. Ao término de cada período de maturação, as amostras foram congeladas a $-18^{0} \mathrm{C}$.

A determinação dos componentes de cor $\mathrm{L}^{*}, \mathrm{a}^{*}$ e $\mathrm{b}^{*}$ nos tempos 0,7 e 14 dias de maturação, foi realizada na superfície das amostras expostas ao ar por 30 minutos após a retirada da embalagem. Foi utilizado o sistema CIE $L^{*} a^{*} b^{*}$, iluminante D65, $10^{0}$ para a observação padrão, onde se utilizou um colorímetro Minolta CR 200b, calibrado para um padrão branco, no qual o $\mathrm{L}^{*}$ representa o índice de luminosidade, variando de $0=$ preto a $100=$ branco; $a^{*}$ intensidade da cor vermelha que varia de verde (-) a vermelho (+); e b* intensidade da cor amarela do azul (-) ao amarelo (+) (HOUBEN et al., 2000). A determinação do croma ( $\left.\mathrm{c}^{*}\right)$ e ângulo de tonalidade $\left(\mathrm{h}^{*}\right)$ foram realizadas de acordo com MacDougal (1994), utilizando-se as informações $L^{*}, a^{*}$ e b*, obtidas nas determinações colorimétricas, com as seguintes fórmulas:

$\mathrm{c}^{*}=\left(\left(\mathrm{a}^{*}\right)^{2}+\left(\mathrm{b}^{*}\right)^{2}\right)^{0,5}$

$\mathrm{h}^{*}=\arctan \left(\mathrm{b}^{*} / \mathrm{a}^{*}\right)$ 
$\mathrm{O}$ pH nos diferentes tempos de maturação foi avaliado com um potenciômetro digital Mettler M1120x, equipado com eletrodo de inserção e resolução de 0,01 unidades.

A CRA nos tempos 0,7 e 14 dias de maturação foi obtida pela diferença de peso de uma subamostra de carne de $2 \mathrm{~g}$ retirada de cada amostra, envolta em papel filtro e acondicionada entre duas placas acrílicas de $64 \mathrm{~cm}^{2}$ e $5 \mathrm{~mm}$ de espessura, antes e depois de ser submetida à pressão de $10 \mathrm{~kg}$, durante cinco minutos, e expressa em porcentagem.

Para a determinação da PPC, a gordura subcutânea da carne foi removida e as amostras foram pesadas. Posteriormente, cada amostra foi colocada em forno elétrico, pré-aquecidos a $180^{\circ} \mathrm{C}$, até atingirem a temperatura interna de $70^{\circ} \mathrm{C}$, quando então foram resfriadas à temperatura ambiente e novamente pesadas. A diferença entre o peso inicial e final, expressa em porcentagem, originou a PPC de cada amostra em cada tempo.

A FC foi avaliada nas mesmas amostras utilizadas para determinação da PPC. Essas amostras foram enroladas em filme plástico e submetidas ao resfriamento a $4^{0} \mathrm{C}$ por 24 horas. Posteriormente, utilizou-se um vazador cilíndrico cortante de $1,27 \mathrm{~cm}$ de diâmetro, acoplado a uma furadeira elétrica, onde extraiu-se de cada amostra resfriada, 10 subamostras na forma de cilindro com diâmetro de $1,27 \mathrm{~cm}$. Para medir a FC cada subamostra foi colocada no sentido das fibras musculares na célula Warner-Bratzler com lâmina $1(0,16 \mathrm{~mm})$ acoplada ao texturômetro MTA.TX. plus com capacidade para $10 \mathrm{~kg}$, integrado ao Software Texture Expert. O texturômetro foi calibrado para velocidade do teste de $200 \mathrm{~mm} / \mathrm{min}$ e pós-teste de $2400 \mathrm{~mm} / \mathrm{min}$ e distância de $40 \mathrm{~mm}$ para cisalhamento completo. Foi considerada a média da FC de 10 subamostras para cada animal em cada tempo.

$\mathrm{O}$ delineamento experimental foi inteiramente casualisado, com três tratamentos e nove repetições. As variáveis de composição centesimal e colesterol foram submetidos a análise de variância usando o PROC GLM do SAS (STATISTICAL ANALYSIS SYSTEM, 1999) e as médias comparadas pelo teste de Tukey com $5 \%$ de probabilidade.

As variáveis FC, CRA, PPC, $\mathrm{pH}$ e cor $\left(L^{*}, a^{*}, b^{*}, c^{*}\right.$ e $\left.h^{*}\right)$ foram analisadas como medida repetida no tempo (dias) no qual se utilizou o PROC MIXED do SAS (STATISTICAL ANALYSIS SYSTEM, 1999), onde considerou-se os efeitos de tratamento, tempo de maturação e a respectiva interação, sendo testadas as estruturas de covariância autoregressiva de $1^{\mathrm{a}}$ ordem (AR1), não estruturada (NU) e simetria composta (CS), e utilizou-se a que obteve o menor valor para o critério de Akaike corrigido (IACC) para cada variável avaliada.

\section{RESULTADOS E DISCUSSÃO}

Não houve diferença entre a composição centesimal, os níveis de colesterol no músculo Longissimus dorsi e na gordura subcutânea $(\mathrm{P}>0,05)$ entre animais suplementados e não suplementados com lipídeos (Tabela 2).

Os resultados semelhantes obtidos para umidade, proteína, cinzas, gordura e colesterol podem ter sido devido as semelhanças no peso de abate, condição sexual, idade e acabamento das carcaças. De acordo com Abrahão et al. (2008), os níveis de proteína na carne apresentam pouca variação, enquanto que a umidade e gordura apresentam comportamos opostos, ou seja, quando 
o teor de gordura é elevado, o teor de umidade é menor e vice-versa. Como não houve diferença entre o grau de acabamento entre os tratamentos $(3,6 ; 3,3$ e $3,0 \mathrm{~mm}$ de EGS para os tratamentos SSL, SOS e SOF respectivamente) era de se esperar pouca diferença na composição centesimal.

Tabela 2. Médias e erros padrões das médias da composição centesimal e colesterol do músculo Longissimus dorsi e colesterol da gordura subcutânea de tourinhos Red Norte suplementados com óleo de fritura e soja terminados em confinamento

\begin{tabular}{lccccc}
\hline \multirow{2}{*}{ Umidade (\%) } & \multicolumn{3}{c}{ Tratamentos } & \multirow{2}{*}{ EPM } & \multirow{2}{*}{ P } \\
\cline { 2 - 5 } & SSL & SOS & SOF & & 0,06 \\
\cline { 2 - 5 } & 72,4 & 73,1 & 73,7 & 0,36 & 0,98 \\
\hline Proteína (\%) & 22,1 & 22,2 & 22,1 & 0,46 & 0,42 \\
Cinzas (\%) & 1,3 & 1,3 & 1,4 & 0,04 & 0,42 \\
Gordura intramuscular (\%) & 2,6 & 2,6 & 2,6 & 0,20 & 0,98 \\
Colesterol Longissimus dorsi (mg/100g) & 33,96 & 32,36 & 31,38 & 2,50 & 0,77 \\
Colesterol gordura subcutânea (mg/100g) & 57,76 & 63,98 & 62,32 & 3,90 & 0,51 \\
\hline Médias seguidas de letras iguais, não diferem entre si (P>0,05) pelo teste de Tukey. & &
\end{tabular}

Médias seguidas de letras iguais, não diferem entre si $(\mathrm{P}>0,05)$ pelo teste de Tukey.

$\mathrm{Na}$ composição centesimal da carne, a gordura é o componente que apresenta maiores variações e, normalmente, sua deposição resulta do balanço energético da dieta e das exigências nutricionais do animal. Entretanto, as suplementações lipídicas nas dietas não foram suficientes para aumentar a deposição de gordura. Isto indica a possibilidade de uso de lipídeos sem, aumentar a proporção de gordura na carne, o que é desejável do ponto de vista alimentar, já que, o consumo excessivo de gorduras saturadas é um fator preponderante no desenvolvimento de doenças cardiovasculares (FERNANDES et al., 2008).

Os valores de colesterol do Longissimus dorsi e da gordura subcutânea não apresentaram diferenças entre tratamentos $(\mathrm{P}>0,05)$. Os valores médios encontrados para o colesterol intramuscular neste trabalho foram inferiores aos valores obtidos por Oliveira et al. (2011) (32,6 vs $49,2 \mathrm{mg} / 100 \mathrm{~g}$ ) utilizando diferentes grãos de oleaginosas (grão de soja, semente de linhaça e caroço de algodão) para bovinos em terminação. Estes resultados demonstram que ao aumentar o nível de EE na dieta não implica, necessariamente, em aumento no nível de colesterol nos diferentes depósitos (gordura intramuscular e subcutânea) da carne, o que demonstra a possibilidade de uso do óleo de soja e óleo de fritura como alternativa de suplementação lipídica, sem alterar o teor de colesterol. Os resultados para as variáveis $\mathrm{FC}$, CRA, PPC e pH estão apresentados na Tabela 3. As médias de efeito principal foram discutidas quando a interação entre tratamentos e dias de maturação foi não significativa $(\mathrm{P}>0,05)$.

Para a FC verificou-se interação significativa $(\mathrm{P}<0,001)$ entre tratamentos e dias durante o processo de maturação. No Tempo 0 (zero) de maturação, a carne dos animais SSL apresentaram os maiores valores para $\mathrm{FC}$, seguida pelo tratamento SOS, e pelo tratamento SOF respectivamente $(\mathrm{P}<0,001)$. Aos 7 dias de maturação não houve diferença $(\mathrm{P}>0,05)$ na $\mathrm{FC}$ entre a carne dos animais SSL e SOS, e estas apresentaram valores superiores $(\mathrm{P}<0,001)$ em relação a carne dos 
animais SOF. Já aos 14 dias de maturação, não houve diferença significativa $(\mathrm{P}>0,05)$ entre as médias dos tratamentos para a FC.

Mesmo o tratamento SSL ter apresentado maior $\mathrm{FC}$ no tempo 0 (zero), ficou evidente que a medida que ocorreu a maturação, a queda da FC foi significativa $(\mathrm{P}<0,01)$ quando comparada aos demais tratamentos. Essa queda na FC pode ter ocorrido devido ao $\mathrm{pH}$ mais baixo da carne, pois de acordo com Koohmaraie (2002), em pH abaixo de 5,7 ocorre o aumento da atividade de enzimas calpaínas ativadas por concentração milimolar de cálcio (mcalpaína). Estas enzimas são ativadas por cálcio livre e são responsáveis pela continuidade do processo de amaciamento, sendo ativadas com aproximadamente 16 horas post-mortem onde permanecem por longos períodos.

Tabela 3. Médias e erros padrões das médias para força de cisalhamento (FC), capacidade de retenção de água (CRA), perda por cozimento (PPC) e pH do músculo Longissimus dorsi de tourinhos Red Norte suplementados com óleo de fritura e soja terminados em confinamento

\begin{tabular}{|c|c|c|c|c|c|c|c|}
\hline \multirow{2}{*}{ Dias } & \multicolumn{3}{|c|}{ Tratamentos $(\mathrm{T})$} & \multirow{2}{*}{ EPM } & \multicolumn{3}{|c|}{ Valores de (P) } \\
\hline & SSL & SOS & SOF & & $\mathrm{T}$ & Dias & T*Dias \\
\hline & & & & $\mathrm{FC}(\mathrm{kgf})$ & & & \\
\hline 0 & $4,50^{\mathrm{Aa}}$ & $3,90^{\mathrm{Ab}}$ & $3,00^{\mathrm{Ac}}$ & 0,214 & $<0,001$ & $<0,001$ & $<0,001$ \\
\hline 7 & $3,80^{\mathrm{Ba}}$ & $3,60^{\mathrm{Aa}}$ & $2,78^{\mathrm{Ab}}$ & 0,214 & $<0,001$ & $<0,001$ & $<0,001$ \\
\hline 14 & $3,20^{\mathrm{Ca}}$ & $3,52^{\mathrm{Aa}}$ & $2,72^{\mathrm{Aa}}$ & 0,214 & $<0,001$ & $<0,001$ & $<0,001$ \\
\hline \multirow[t]{2}{*}{ Média } & 3,83 & 3,67 & 2,80 & & & & \\
\hline & & & & $\mathrm{CRA}(\%)$ & & & \\
\hline 0 & $73,63^{\mathrm{Ac}}$ & $75,53^{\mathrm{Ab}}$ & $77,61^{\mathrm{Aa}}$ & 0,800 & $<0,01$ & 0,79 & 0,96 \\
\hline 7 & $73,32^{\mathrm{Ac}}$ & $74,90^{\mathrm{Ab}}$ & $77,50^{\mathrm{Aa}}$ & 0,800 & $<0,01$ & 0,79 & 0,96 \\
\hline 14 & $72,86^{\mathrm{Ac}}$ & $75,21^{\mathrm{Ab}}$ & $77,43^{\mathrm{Aa}}$ & 0,800 & $<0,01$ & 0,79 & 0,96 \\
\hline \multirow[t]{2}{*}{ Média } & 73,27 & 75,21 & 77,51 & & & & \\
\hline & & & & PPC (\%) & & & \\
\hline 0 & $30,53^{\mathrm{Aa}}$ & $25,08^{\mathrm{Ab}}$ & $20,85^{\mathrm{Ac}}$ & 1,250 & $<0,001$ & 0,07 & 0,26 \\
\hline 7 & $30,60^{\mathrm{Aa}}$ & $24,05^{\mathrm{Ab}}$ & $20,08^{\mathrm{Ac}}$ & 1,250 & $<0,001$ & 0,07 & 0,26 \\
\hline 14 & $30,23^{\mathrm{Aa}}$ & $26,81^{\mathrm{Ab}}$ & $21,68^{\mathrm{Ac}}$ & 1,250 & $<0,001$ & 0,07 & 0,26 \\
\hline \multirow[t]{2}{*}{ Média } & 30,45 & 25,31 & 20,87 & & & & \\
\hline & & & & $\mathrm{pH}$ & & & \\
\hline 0 & $5,56^{\mathrm{Ac}}$ & $5,87^{\mathrm{Ab}}$ & $6,34^{\mathrm{Aa}}$ & 0,095 & $<0,001$ & 0,30 & 0,17 \\
\hline 7 & $5,50^{\mathrm{Ac}}$ & $5,83^{\mathrm{Ab}}$ & $6,27^{\mathrm{Aa}}$ & 0,095 & $<0,001$ & 0,30 & 0,17 \\
\hline 14 & $5,46^{\mathrm{Ac}}$ & $5,83^{\mathrm{Ab}}$ & $6,27^{\mathrm{Aa}}$ & 0,095 & $<0,001$ & 0,30 & 0,17 \\
\hline Média & 5,47 & 5,84 & 6,30 & & & & \\
\hline
\end{tabular}

Letras iguais minúsculas na mesma linha e maiúsculas na mesma coluna, não diferem entre si $(\mathrm{P}>0,05)$ pelo teste de Tukey.

Dessa forma, a suplementação lipídica implicaria em menor tempo de maturação da carne, que além de diminuir os custos com refrigeração, permitiria também maior quantidade de carcaça armazenada em um menor intervalo de tempo, o que otimizaria a utilização das câmaras frias.
Além disso, a menor FC nos tempos 0 e 7 dias de maturação da carne dos animais SOF em relação aos outros tratamentos pode estar associada ao $\mathrm{pH}$ mais elevado, maior CRA $(\mathrm{P}<0,01)$ que via de regra, resulta em menores perdas de umidade, bem como, as menores PPC $\quad(\mathrm{P}<0,001)$ Juntas estas 
características impactam positivamente na suculência e na maciez da carne.

Houve diferença para a CRA entre os tratamentos $(\mathrm{P}<0,01)$. $\mathrm{O}$ tratamento com óleo de fritura apresentou os maiores valores, seguidos pelos tratamentos com inclusão de óleo de soja e sem inclusão lipídica respectivamente.

A carne com maior CRA dos animais SOF ocorreu possivelmente devido ao elevado $\mathrm{pH}(6,3)$. Segundo Souza et al. (2004) quando o pH diminui pouco após as primeiras horas de abate e permanece acima de 6,0 completadas 24 hora post mortem, a carne apresenta elevada CRA. Ainda segundo Koohmaraie (2002), a formação de ácido lático e a redução do $\mathrm{pH}$ durante a glicólise post mortem são responsáveis pela diminuição da capacidade da carne em reter água durante a aplicação de forças como cortes e aquecimento. Em pH 5,2 - 5,3 (ponto isoelétrico das proteínas musculares), a carne apresenta menor CRA. Em $\mathrm{pH}$ acima de 5,5 existe excesso de cargas negativas que determinam repulsão dos filamentos protéicos, o que permite maior espaço para as moléculas de água e reduz a força mecânica necessária para o corte e as perdas durante o cozimento, o que explicaria os menores valores de CRA para o tratamento SSL.

Houve diferença para as PPC entre os tratamentos $(\mathrm{P}<0,001)$. $\mathrm{O}$ tratamento SSL apresentou os maiores valores, seguidos pelos tratamentos com SOS e SOF respectivamente.

As maiores PPC observadas para a carne dos animais SSL e SOS, pode estar associada aos menores valores de $\mathrm{pH}$. O pH mais baixo se relaciona a uma maior perda de água e que pode influenciar as perdas durante o processo de cozimento. De acordo com Souza et al. (2004), a avaliação da qualidade de carne numa faixa de $\mathrm{pH}$ variando de 5,6 a 7,0 mostram que a PPC diminui linearmente com o aumento do $\mathrm{pH}$. Fato observado no presente estudo, pois o tratamento que apresentou os maiores valores de $\mathrm{pH}$ (óleo de fritura) apresentou a menor PPC, enquanto que o tratamento SSL apresentou menores valores de $\mathrm{pH}$ e maiores valores de PPC.

O tempo de maturação não influenciou o $\mathrm{pH}$ da carne nos diferentes tratamentos avaliados $(\mathrm{P}>0,05)$. Entretanto, o $\mathrm{pH}$ da carne dos animais SOF foi superior $(\mathrm{P}<0,001)$ ao $\mathrm{pH}$ da carne dos animais SOS e SSL. Apesar do $\mathrm{pH}$ da carne dos animais SOS e SSL serem diferentes entre si $(\mathrm{P}<0,001)$, os mesmos encontram-se em patamares considerados adequados para manutenção da qualidade da carne. Todavia, o elevado $\mathrm{pH}$ do tratamento com suplementação com óleo de fritura $(6,3)$, indica carne tipo $D F D$ $(\mathrm{pH}>6,0)$ o que compromete seu valor comercial.

Estes resultados divergem dos resultados observados na literatura em trabalhos com animais confinados avaliando diferentes fontes e níveis de suplementação lipídica (NELSON et al., 2008; OLIVEIRA et al., 2011), onde não houve ocorrência de carne tipo DFD.

Os valores médios para as coordenadas de cor $\left(L^{*}, a^{*}\right.$ e $\left.b^{*}\right)$, índice de saturação $\left(\mathrm{c}^{*}\right)$ e ângulo de tonalidade $\left(\mathrm{h}^{*}\right)$ para cada tratamento em função dos tempos de maturação estão apresentados na Tabela 4. As médias de efeito principal foram discutidas quando a interação entre tratamentos e dias de maturação foram não significativas $(\mathrm{P}>0,05)$.

Os valores encontrados para as coordenadas de cor foram influenciados $(\mathrm{P}<0,001)$ pelos tratamentos. Verificouse também influência $(\mathrm{P}<0,001)$ de dias de maturação para a $\mathrm{a}^{*} \mathrm{e}$ interação entre tratamento e dias de maturação para $b^{*}$, $h^{*}(\mathrm{P}<0,001)$ e c* $(\mathrm{P}<0,05)$. 
Tabela 4. Médias e erros padrões das médias para luminosidade $\left(\mathrm{L}^{*}\right)$, intensidade da cor vermelha $\left(a^{*}\right)$, intensidade da cor amarela $\left(b^{*}\right)$, índice de saturação $\left(c^{*}\right)$ e ângulo de tonalidade $\left(\mathrm{h}^{*}\right)$ do músculo Longissimus dorsi de tourinhos Red Norte suplementados com óleo de fritura e soja terminados em confinamento

\begin{tabular}{|c|c|c|c|c|c|c|c|}
\hline \multirow{2}{*}{ Dias } & \multicolumn{3}{|c|}{ Tratamentos $(\mathrm{T})$} & \multirow{2}{*}{ EPM } & \multicolumn{3}{|c|}{ Valores de $(\mathrm{P})$} \\
\hline & SSL & SOS & SOF & & $\mathrm{T}$ & Dias & T*Dias \\
\hline & \multicolumn{7}{|c|}{$\mathrm{L}^{*}$} \\
\hline 0 & $37,47^{\mathrm{Aa}}$ & $34,39^{\mathrm{Ab}}$ & $30,60^{\mathrm{Ac}}$ & 0,965 & $<0,001$ & 0,66 & 0,06 \\
\hline 7 & $37,76^{\mathrm{Aa}}$ & $35,20^{\mathrm{Ab}}$ & $30,75^{\mathrm{Ac}}$ & 0,965 & $<0,001$ & 0,66 & 0,06 \\
\hline 14 & $39,35^{\mathrm{Aa}}$ & $35,28^{\mathrm{Ab}}$ & $30,85^{\mathrm{Ac}}$ & 0,965 & $<0,001$ & 0,66 & 0,06 \\
\hline \multirow[t]{2}{*}{ Médias } & 38,19 & 34,96 & 31,07 & & & & \\
\hline & \multicolumn{7}{|c|}{$a^{*}$} \\
\hline 0 & $18,50^{\mathrm{Ba}}$ & $15,61^{\mathrm{Bb}}$ & $14,83^{\mathrm{Bb}}$ & 0,563 & $<0,001$ & $<0,001$ & 0,30 \\
\hline 7 & $20,65^{\mathrm{Aa}}$ & $18,06^{\mathrm{Ab}}$ & $16,88^{\mathrm{Ac}}$ & 0,563 & $<0,001$ & $<0,001$ & 0,30 \\
\hline 14 & $21,12^{\mathrm{Aa}}$ & $18,59^{\mathrm{Ab}}$ & $16,60^{\mathrm{Ac}}$ & 0,563 & $<0,001$ & $<0,001$ & 0,30 \\
\hline \multirow[t]{2}{*}{ Médias } & 20,09 & 17,42 & 16,10 & & & & \\
\hline & \multicolumn{7}{|c|}{$\mathrm{b}^{*}$} \\
\hline 0 & $3,03^{\mathrm{Ca}}$ & $1,19^{\mathrm{Bb}}$ & $1,12^{\mathrm{Ab}}$ & 0,607 & $<0,001$ & $<0,001$ & $<0,001$ \\
\hline 7 & $5,54^{\mathrm{Ba}}$ & $3,37^{\mathrm{Ab}}$ & $0,97^{\mathrm{Ac}}$ & 0,607 & $<0,001$ & $<0,001$ & $<0,001$ \\
\hline 14 & $7,94^{\mathrm{Aa}}$ & $3,93^{\mathrm{Ab}}$ & $1,28^{\mathrm{Ac}}$ & 0,607 & $<0,001$ & $<0,001$ & $<0,001$ \\
\hline \multirow[t]{2}{*}{ Médias } & 5,50 & 2,83 & 0,79 & & & & \\
\hline & \multicolumn{7}{|c|}{$\mathrm{c}^{*}$} \\
\hline 0 & $18,75^{\mathrm{Ba}}$ & $15,73^{\mathrm{Bb}}$ & $14,87^{\mathrm{Bb}}$ & 0,637 & $<0,001$ & $<0,001$ & 0,04 \\
\hline 7 & $21,40^{\mathrm{Aa}}$ & $18,50^{\mathrm{Ab}}$ & $17,01^{\mathrm{Ac}}$ & 0,637 & $<0,001$ & $<0,001$ & 0,04 \\
\hline 14 & $22,57^{\mathrm{Aa}}$ & $19,17^{\mathrm{Ab}}$ & $17,67^{\mathrm{Ac}}$ & 0,637 & $<0,001$ & $<0,001$ & 0,04 \\
\hline \multirow[t]{2}{*}{ Médias } & 20,91 & 17,80 & 16,18 & & & & \\
\hline & \multicolumn{7}{|c|}{$\mathrm{h}^{*}$} \\
\hline 0 & $9,36^{\mathrm{Ca}}$ & $3,91^{\mathrm{Bb}}$ & $0,12^{\mathrm{Ac}}$ & 1,776 & $<0,001$ & $<0,001$ & $<0,001$ \\
\hline 7 & $15,28^{\mathrm{Ba}}$ & $9,72^{\mathrm{Ab}}$ & $3,02^{\mathrm{Ac}}$ & 1,776 & $<0,001$ & $<0,001$ & $<0,001$ \\
\hline 14 & $21,54^{\mathrm{Aa}}$ & $11,02^{\mathrm{Ab}}$ & $4,27^{\mathrm{Ac}}$ & 1,776 & $<0,001$ & $<0,001$ & $<0,001$ \\
\hline Médias & 15,39 & 8,22 & 2,47 & & & & \\
\hline
\end{tabular}

Letras iguais minúsculas na mesma linha e maiúsculas na mesma coluna, não diferem entre si $(\mathrm{P}>0,05)$ pelo teste de Tukey.

As médias de $\mathrm{L}^{*}$ foram superiores durante $o$ período de maturação $(\mathrm{P}<0,001)$ para o tratamento $\mathrm{SSL}$, o que indica uma cor mais clara para a carne deste tratamento, em relação à carne dos animais suplementados com lipídios. Já o tratamento SOF apresentou menor luminosidade $(\mathrm{P}<0,001)$, o que resultou em uma coloração mais escura, o que está diretamente relacionado com o $\mathrm{pH}$ e CRA mais elevados da carne.

Para a variável $\mathrm{a}^{*}$, as médias foram superiores $(\mathrm{P}<0,001)$ para o tratamento $\mathrm{SSL}$, em todos os tempos de maturação avaliados, o que indicou maior intensidade da cor vermelha, em comparação à carne dos demais tratamentos. Entre os animais suplementados com lipídeos, aqueles que receberam dieta com óleo de fritura, apresentaram menor intensidade da cor vermelha $(\mathrm{P}<0,001)$ aos 7 e 14 dias de maturação, embora tenham apresentado uma cor vermelha semelhante $(\mathrm{P}>0,05)$ no dia 0 (zero) de maturação.

A variação na intensidade $a^{*}$ observada pode estar relacionada ao estado da forma química dos pigmentos heme que dependendo de seu estado de oxidação ou redução e a substância ligante na posição 6 de sua estrutura (geralmente $\mathrm{O}_{2}$ e $\mathrm{H}_{2} 0$ ) produzirá proporções variáveis de mioglobina reduzida, oximioglobina e metamioglobina, que 
afeta as características espectrais, e portanto, a cor da carne. A maior intensidade de $a^{*}$ observada para a carne dos animais SSL decorre, possivelmente, de uma maior proporção de oximioglobina, de cor vermelha cereja, em relação às outras formas de mioglobina presentes na carne, aliada a um menor grau de oxidação desse pigmento.

Para a variável $b^{*}$, a carne dos animais submetidos aos tratamentos com lipídios no tempo 0 (zero) de maturação, não diferiram entre si $(\mathrm{P}>0,05)$ e foram inferiores $(\mathrm{P}<0,001)$ ao tratamento SSL. Aos 7 dias de maturação, a carne dos animais em todos os tratamentos foram diferentes entre si $(\mathrm{P}<0,001)$, com o mesmo comportamento até os 14 dias, onde tratamento SSL apresentou os maiores valores $(\mathrm{P}<0,001)$ seguido pelos tratamentos SOS e SOF respectivamente.

Para a carne dos animais do tratamento SSL, verificou-se que os valores de $b^{*}$ foram diferentes $(\mathrm{P}<0,001)$ ao longo do tempo de maturação. Já para o tratamento SOS no dia 0 (zero) e 7 de maturação houve diferença significativa $(\mathrm{P}<0,001)$ para $\mathrm{b}^{*}$, embora não tenha ocorrido diferença entre os dias 7 e 14 $(\mathrm{P}>0,05)$. Entretanto, para o tratamento $\mathrm{SOF}$, não foi observado diferença $(\mathrm{P}>0,05)$, ao longo do tempo de maturação. Esse resultado difere dos resultados observados em carne fresca (FERNANDES et al., 2008; NELSON et al., 2008; OLIVEIRA et al., 2011), avaliando diferentes fontes lipídicas em dietas de bovinos em terminação.

A variação na intensidade $d a b^{*}$ observada pode estar relacionada à composição e quantidade de carotenoides presentes nas dietas. A carne dos animais SOS e SOF apresentaram menor intensidade de $\mathrm{b}^{*}$ em relação à carne dos animais SSL, provavelmente devido à maior quantidade de pigmentos naturais (carotenos e xantofila) presentes na dieta SSL. Uma fração do milho da dieta foi substituída por lipídios o que possivelmente, aportaram menores quantidades destes carotenoides na carne. O óleo de fritura apresenta sua composição e estado de oxidação muito variáveis em função de tempo e temperatura aos quais o óleo foi submetido e o tipo de alimento utilizado no processo de fritura. Já o óleo de soja, por outro lado, é submetido a um processo industrial de refinamento $\mathrm{e}$ clareamento no qual grande parte dos pigmentos e carotenos são removidos (DORSA, 2004).

Outro aspecto a considerar é que em ambiente de baixa pressão de oxigênio, como na embalagem a vácuo, as xantofilas presentes no milho, são consideradas absorvedores de oxigênio entre os carotenoides naturais, se constituindo, em um potente antioxidante, que pode ter contribuído para a absorção de oxigênio remanescente na embalagem, e preservou melhor o estado químico dos carotenos absorvidos e presentes na carne (SILVA et al., 2010), o que explicaria os maiores valores de $b^{*}$ para a carne dos animais SSL.

As médias de $\mathrm{c}^{*}$ foram semelhantes $(\mathrm{P}>0,05)$ entre os tratamentos que receberam suplementação lipídica no dia 0 (zero), e menor $(\mathrm{P}<0,001)$ em relação ao tratamento SSL. Aos 7 e 14 dias de maturação as médias de $\mathrm{c}^{*}$ diferiram entre si $(\mathrm{P}<0,001)$ para todos os tratamentos, onde o tratamento SSL apresentou as maiores médias $(\mathrm{P}<0,001)$ seguidas pelos tratamentos SOS e SOF respectivamente. No dia 0 (zero) de maturação, a carne dos animais SSL apresentou menores médias de $\mathrm{c}^{*}$ $(\mathrm{P}<0,001)$ em relação aos 7 dias, que por sua vez, não diferiram entre si $(\mathrm{P}>0,05)$ aos 14 dias, sendo observado o mesmo comportamento para os demais tratamentos. 
Ao longo da maturação os parâmetros de $c^{*}$ evoluíram de forma semelhante entre os tratamentos, semelhantes aos comportamentos observados para $\mathrm{a}^{*} \mathrm{e}$ $b^{*}$. O maior valor de $c^{*}$ observado no tratamento SSL indica maior índice de saturação de cor na carne desses animais, em relação aos demais, fato explicado pela proporção e tipo de mioglobina presente na carne dos animais de cada tratamento. Quando se avaliou individualmente cada tratamento ao longo do tempo de maturação, verificou-se maior intensidade de saturação da cor a partir de 7 dias para todos os tratamentos.

As médias de $\mathrm{h}^{*}$ foram diferentes entre si $(\mathrm{P}<0,001)$ em todos os tratamentos nos tempos 0,7 e 14 dias de maturação, com maiores valores para o tratamento SSL, seguido pelo tratamento SOS e SOF respectivamente. Para o tratamento SSL verificou-se vermelho mais brilhante com valores crescentes para $\mathrm{h}^{*}(\mathrm{P}<0,001)$ ao longo do tempo de maturação. Já para o tratamento SOS verificou-se aumento significativo $(\mathrm{P}<0,001)$ das médias de $\mathrm{h} *$ de 0 (zero) para 7 dias de maturação, embora, de 7 para 14 dias não ter encontrado diferença. Por outro lado, para o tratamento SOF, não foi observado diferença $(\mathrm{P}>0,05)$, ao longo do tempo de maturação. $O$ índice de tonalidade (h*) apresentou comportamento semelhante à intensidade da cor amarela (b*). Além disso, verificou-se um aumento do índice de saturação $\left(\mathrm{c}^{*}\right)$ da cor ao longo do tempo de maturação para todos os tratamentos. Como houve aumentos de $\mathrm{h}^{*}$ para a carne dos animais SSL ao longo da maturação e dos animais com SOS a partir de 7 dias, pode-se dizer que a predominância de vermelho nesses casos diminuiu $\mathrm{e}$ aumentou a do amarelo, com a aproximação da tonalidade laranja.
A suplementação com lipídios não influenciou a composição centesimal da carne e dos teores de colesterol. O óleo de soja influenciou a cor da carne, sem, contudo, comprometer sua qualidade. Por outro lado, o elevado $\mathrm{pH}$ da carne dos animais suplementados com óleo de fritura, produziu uma carne $D F D$, indesejável para o consumo humano. Já a maturação reduziu a força de cisalhamento da carne dos animais não suplementados com lipídeos, porém não apresentou melhora nos animais suplementados. Assim, mais estudos com óleo de fritura necessitam ser feito para evidenciar a verdadeira influencia deste resíduo sobre o processo de acidificação muscular no post mortem.

\section{REFERÊNCIAS}

ABRAHÃO, J.J.S.; MARQUES, J.A.; MACEDO, L.M.; PRADO, J.M.; VISANTAINER, J.V.; PRADO, I.N. Physical-chemical composition and fatty acid profile in Longissimus muscle of young bulls from different genetic groups finished in feedlot. Acta Scientiarum.

Animal Science, v.30, p.443-449, 2008.

ASSOCIATION OF OFFICIAL ANALYTICAL CHEMISTS - AOAC. Official Methods of Association of Official Analytical Chemist. 18.ed. Gaithersburg, 2005. 1298p.

BRAGAGNOLO, N.; RODRIGUEZAMAYA, D.B. Índices de colesterol em carne suína e bovina e efeito do cozimento. Ciência e Tecnologia de Alimentos, n.15, p.11-17, 1995.

BRASIL. MINISTÉRIO DA AGRICULTURA. Regulamento da inspeção industrial e sanitária de produtos de origem animal. Brasília, 1997. 154p. 
DORSA, R. Tecnologia de óleos vegetais. Campinas: Ideal, 2004. 463p.

FERNANDES, A.R.M.; SAMPAIO, A.A.M.; HENRIQUE, W.; OLIVEIRA, E.A.; TULLIO, R.R.; PERECIN, D. Carcass and meat characteristic of cattle receiving differents diets in feedlot..

Arquivo Brasileiro de Medicina Veterinária e Zootecnia, v.60, p.139147, 2008.

FOLCH, J.; LESS,M.; STANLEY, S. A Simple method for the isolation and purification of total lipids from animal tissues. Journal of Biological Chemistry, v.226, p.497-509, 1957.

HOUBEN, J.H.; Van DIJK, A.; EIKELENBOOM, G.; HOVINGBOLINK, A.H. Effect of dietary vitamin E supplementation, fat level and packaging on color stability and lipid oxidation in minced beef. Meat Science, v.55, p.331-336, 2000.

KOOHMARAIE, M.; KENT, M.P.; SHACKEIFORD, S.D.; VEISETH, E.; WHEELER, T.L. Meat tenderness and muscle growth: is there any relationship? Meat Science, v.62, p.345-352, 2002.

MacDOUGAL, D.B. Color meat. In: PEARSON, A.M.; DUTSON, T.R. (Ed.). Quality attributes and their measurement in meat, poultry and fish products: advances in meat research series. London: Blackie Academic \& Professional, 1994. v.9, p.79-93.

NATIONAL RESEARCH COUNCIL NRC. Nutrient Requirements of beef cattle. Washington: National Academy Press, 2000. 242p.

NELSON, M.L.; BUSBOOM, J.R.; ROSS, C.F.; O'FALLON, J.V. Effects of supplemental fat on growth performance and quality of beef from steers fed corn finishing diets. Journal of Animal

Science, v.86, p.936-948, 2008.

OLIVEIRA, D.M.; LADEIRA, M.M.; CHIZZOTTI, M.L.; MACHADO NETO O.R.; RAMOS, E.M.; GONÇALVES, T.M.; BASSI, M.; LANNA, D.P.D.; RIBEIRO, J.S. Fatty acid profile and qualitative characteristics of meat from zebu steers fed with different oilseeds.

Journal of Animal Science, v.89, p.2546-2555, 2011.

RODRIGUES FILHO, M.; ANDRADE, I.F.; LADEIRA, M.M.; RODRIGUES, N.E.B.; LOPES, L.S. Characteristics of carcass and commercial meat cuts of Red Norte young bulls supplemented with fry and soybeans oil finished in feedlot..

Revista Brasileira de Saúde e Produção Animal [online], v.14, p.54-66, 2013.

STATISTICAL ANALYSIS SYSTEM.. Procedures guide of computers. 6th ed. New York, 1999. v.3, 373p.

SILVA, M.L.C.; COSTA, R.S.; SANTANA, A.S.; KOBLITZ, M.G.B. Phenolic compounds, carotenoids and antioxidant activity in plant products.

Semina: Ciências Agrárias, v.31, p.669$682,2010$.

SOUZA, X.R.; BRESSAN, M.C.; PÉREZ, J.R.O.; FARIA, P.B.; VIEIRA, J.O.; KABEYA, D.M. Effects of breed group, gender and slaughter weight group on the physical-chemical parameter of the growing lambs. Ciência e Tecnologia dos Alimentos, v.24, p.543-549, 2004.

Data de recebimento: 07/09/2013

Data de aprovação: 21/02/2014 